\title{
The neutrino velocity anomaly as an explanation of the missing observation of neutrinos in coincidence with GRB
}

\author{
D. Autiero ${ }^{a}$ P. Migliozzi ${ }^{b}$ A. Russo ${ }^{b}$ \\ a IPNL, Université Claude Bernard Lyon I, CNRS/IN2P3, F-69622 Villeurbanne, \\ France \\ ${ }^{\mathrm{b}}$ I.N.F.N., Sezione di Napoli, Complesso Universitario Monte Sant'Angelo, \\ I-80125, Naples, Italy
}

\begin{abstract}
The search for neutrinos emitted in coincidence with Gamma-Bay Burst has been so far unsuccessfully. In this paper we show that the recent result reported by the OPERA Collaboration on an early arrival time of muon neutrinos with respect to the one computed assuming the speed of light in vacuum could explain the null search for neutrinos in coincidence with Gamma-Ray Burst.
\end{abstract}




\section{Introduction}

Gamma-Bay Burst (GRB) are among the most violent events in the Universe and could be one of the possible source of ultra-high energy cosmic-rays. The currently leading model for GRBs production is the fireball model $[1,2]$. Schematically, it explains the emission of GRBs by considering an energy source (central engine) consisting of a newly formed black hole that rapidly increases its mass. Within the fireball model framework, observed $\gamma$-rays are produced by synchrotron emission of electrons accelerated to high energy by internal shocks within the expanding wind. In addition to electrons, protons are also expected to be accelerated through the Fermi mechanism and may interact with the $\mathrm{keV}-\mathrm{MeV}$ photons forming a $\Delta^{+}$resonance that decays into pions. From the subsequent pion decay a detectable flux of high-energy neutrinos with energy around $10^{14} \mathrm{eV}$ is expected to be emitted in coincidence with GRBs. The production neutrino flavor ratio expected from this model is $\left(\nu_{e} ; \nu_{\mu} ; \nu_{\tau}\right)=(1 ; 2 ; 0)$. However, due to the neutrino oscillation mechanism this ratio is changed into $(1 ; 1 ; 1)[3,4]$. According to the fireball model a detector of $\sim 1 \mathrm{~km}^{2}$ should detect about 10-100 muonic neutrino interactions per year in coincidence with GRB. On top of the fireball approach, there are other models that describe the GRB production. It is worth mention that they also

demand production of high-energy neutrinos in coincidence with GRBs see $[5,6]$ and references therein.

This Letter is organized as follow: after a brief review of the search for high-energy neutrinos in coincidence with GRBs, we summarize the recent results published by the OPERA Collaboration [7] that show a neutrino speed faster than the speed of light with a statistical significance of $6.0 \sigma$. Finally, we discuss the impact of the OPERA results on the search for neutrino emission from GRBs.

\section{Present status of the search for neutrinos emission from Gamma-Ray Bursts}

The IceCube Neutrino Observatory is a neutrino telescope constructed at the AmundsenScott South Pole Station in Antarctica[8]. Similar to its predecessor, the Antarctic Muon And Neutrino Detector Array (AMANDA) [9], IceCube contains thousands of spherical optical sensors called Digital Optical Modules (DOMs), each with a photomultiplier tube (PMT) and a single board data acquisition computer which sends digital data to the counting house on the surface above the array IceCube was completed on the 18th December, 2010 see [10].

The main goal of IceCube is to detect point sources of neutrinos that could help explain the mystery of the origin of the highest energy cosmic rays. Astrophysical events which are cataclysmic enough to create such high energy particles would probably also create high energy neutrinos, which could travel to the Earth with very little deflection, because neutrinos interact so rarely. IceCube could observe these neutrinos: its 
observable energy range is about $100 \mathrm{GeV}$ to several $\mathrm{PeV}$.

As explained in Section 1 when protons collide with one another or with photons, the result is usually pions. Charged pions decay into muons and muon neutrinos whereas neutral pions decay into gamma rays. Potentially, the neutrino flux and the gamma ray flux may coincide in certain sources such as gamma ray bursts and supernova remnants, indicating the elusive nature of their origin. Therefore, the IceCube Collaboration performed a dedicated search for neutrino emission from GRB sources.

The search discussed in [11] has been conducted as follow. A catalog of GRB observed in the Northern Emisphere has been assembled via the GRB coordinates Network (GCN) [12]. A search for possible neutrino signal has been performed in a time window around the observed GRB. From April 2008 to May 2009 IceCube was operating with 40 out of the 86 strings composing the whole detector. During this period 129 GRBs were observed in the Northern Emisphere. Two different analyses have been conducted to exploit this sample of GRBs. One dedicated to the search for neutrinos produced in $p-\gamma$ interactions in the prompt phase of the GRB fireball (model dependent analysis); a second more generic search for any neutrino emission from GRB over a wide range of energies and emission times (model independent analysis). Both searches gave a null results for neutrino emission in coincidence with GRBs. The IceCube Collaboration in the conclusions of [11] wrote While the specific neutrino-flux predictions of the fireball model provided by Waxman and Bahcall [1] and by Guetta et al. [2] are excluded (90\% confidence) by this work, we have not yet ruled out the general picture of fireball phenomenology. The neutrino flux we compute for GRBs is determined by the flux of protons accelerated in the fireball, and by the fraction of proton energy transferred to charged pions $\left(f_{\pi}\right)$. The proton flux can be chosen either such that the energy in gammas and protons is equal or set to the flux of cosmic rays above $10^{18} \mathrm{eV}$, with similar results. $f_{\pi}$ is determined largely by assuming protons are accelerated, in conjunction with the observed low optical thickness of the source. Due to uncertainties in the bulk boost factor and internal structure of the shocks, $f_{\pi}$ may range from $10-30 \%$ [13], causing an uncertainty of about a factor of 2 on our calculation of the flux, which used $f_{\pi} \sim 0.2$. Future observations by IceCube will push our sensitivity below the level of this theoretical uncertainty on $f_{\pi}$ and allow direct constraints on acceleration of protons to ultra-high energies in Gamma Ray Bursts. Recently preliminary results from a search with 59 out of the 86 strings composing the whole detector has been presented [14]. No neutrinos have been observed in coincidence with a GRB, while 8 events were expected.

\section{Impact of neutrino velocity measurement on GRB neutrino searches}

The OPERA neutrino experiment [15] in the underground Gran Sasso Laboratory (LNGS) was designed to perform the first detection of neutrino oscillations in direct appearance mode in the $\nu_{\mu} \rightarrow \nu_{\tau}$ channel channel, the $\nu_{\tau}$ signature being the identification of the $\tau$ lepton created in its charged current (CC) interaction [16]. A precision 
measurement of the neutrino velocity by using muonic neutrinos from the CNGS beam [17] has been reported in [REF]. This measurement was made possible thanks to the high-statistics data taken by the OPERA detector, by dedicated upgrades of the CERN timing system for the time tagging of the CNGS beam and of the OPERA detector resulting in a reduction of the systematic uncertainties down to the level of the statistical error ( $\sim 10 \mathrm{~ns})$. Finally, by a high-accuracy geodesy campaign that allowed measuring the $730 \mathrm{~km}$ CNGS baseline with a precision of the order of $20 \mathrm{~cm}$. All the details are given in [7] and references therein.

Having defined $\delta t$ as the difference between $T O F_{c}$ (the expected time of flight from the neutrino source to the detector assuming the speed of light) and $T O F_{\nu}$ (the time of flight of CNGS neutrinos from the neutrino source to the detector), i.e. $\delta t=T O F_{c}-$ $T O F_{\nu}$, the final result on the measurement is

$$
\delta t=(60.7 \pm 6.9(\text { stat. }) \pm 7.4(\text { sys. })) \mathrm{ns} .
$$

Namely, the measurement indicates an early arrival time of CNGS muon neutrinos with respect to a light signal with a $6 \sigma$ significance.

How this result affects the search for neutrinos in coincidence with GRBs? Here, we do not want to attempt theoretical interpretations of the result on neutrino velocity, i.e. is there an energy dependence of the neutrino velocity? Therefore, let's assume conservatively that the anticipation measured by OPERA is constant also at higher neutrino energies.

Typically, the distance from where a GRB originated is measured in terms of the quantity $z$, i.e. the source read-shift, and $z=1$ corresponds to a distance of $1.302773 \times$ $10^{+23} \mathrm{~km}$. In the sample of GRBs analyzed by the IceCube Collaboration the average $z$ is about 2 and ranges between 0.8 and 2.5 [11].

If we assume that every $730 \mathrm{~km}$ the neutrinos anticipate by $60 \mathrm{~ns}$ the light, i.e. no energy dependence is assumed, we can compute the anticipation of the neutrinos arrival time with respect to the light as a function of $z$. The results are shown in Table 1. Even for the closest GRBs the neutrino arrival time is anticipated, with respect to the light arrival time, by more than hundred thousand years! Therefore, this result could explain why the specific neutrino-flux predictions of the fireball model provided by Waxman and Bahcall [1] and by Guetta et al. [2] are excluded (90\% confidence) by the IceCube experiment. Nevertheless, the general picture of the fireball phenomenology is not yet ruled out.

The forthcoming results of the IceCube Collaboration on the search for neutrinos from GRB are, therefore, of the outmost importance. Indeed, a null result would further exclude the neutrino-flux predictions of the fireball model. Such a result could then be explained either by a complete failure of the fireball model or by advocating new phenomena. Unfortunately, neutrino telescopes will be never able to measure an earlier time arrival of neutrinos from GRB, but they could only give indirect support to the 
OPERA result. On the other hand, an unambiguous detection of neutrino from GRBs would disprove the interpretation of the OPERA result in terms of a neutrino traveling faster than light.

\begin{tabular}{|c|c|c|c|}
\hline$z$ & $\mathrm{Mpc}$ & Neutrino anticipation $\left(10^{10} \mathrm{~s}\right)$ & Neutrino anticipation $\left(10^{5}\right.$ year $)$ \\
\hline 0,5 & 1657,67 & 417,73 & 2,65 \\
\hline 1 & 3315,33 & 835,46 & 4,22 \\
\hline 2 & 5275,05 & 1329,31 & 5,90 \\
\hline 4 & $\mathrm{~h} 7383,55$ & 1860,65 & 7,41 \\
\hline
\end{tabular}

Table 1

Anticipation of the neutrinos arrival time with respect to the light as a function of $z$.

\section{References}

[1] E. Waxman, J. N. Bahcall, Phys. Rev. Lett. 78 (1997) 2292-2295. astro-ph/9701231.

[2] D. Guetta, D. Hooper, J. Alvarez-Muniz, F. Halzen, E. Reuveni, Astropart. Phys. 20 (2004) 429-455. astro-ph/0302524.

[3] J. G. Learned, S. Pakvasa, Astropart. Phys. 3 (1995) 267-274. hep-ph/9405296, hep-ph/9408296.

[4] H. Athar, C. S. Kim, J. Lee, Mod. Phys. Lett. A21 (2006) 1049-1066. hep-ph/0505017.

[5] J. K. Becker, F. Halzen, A. O Murchadha, M. Olivo, Astrophys. J. 721 (2010) 18911899. arXiv:0911.2202 [astro-ph.HE]].

[6] S. Gao, K. Toma, P. Meszaros, Phys. Rev. D83 (2011) 103004. arXiv:1103.5477 [astroph.HE]].

[7] T. Adam et al., "Measurement of the neutrino velocity with the OPERA detector in the CNGS beam ," arXiv:1109.4897 [hep-ex]].

[8] F. Halzen, S. R. Klein, Rev. Sci. Instrum. 81 (2010) 081101. arXiv:1007.1247 [astroph.HE]].

[9] E. Andrés et al. [AMANDA Collaboration], Nature 410 (2001) 441.

[10] IceCube Collaboration, material available at http://www.icecube.wisc.edu

[11] IceCube Collaboration, R. Abbasi et al., Phys. Rev. Lett. 106 (2011) 141101. arXiv:1101.1448 [astro-ph.HE]].

[12] "GRB Coordinates Netweork", http://gcn.gsfc.nasa.gov/.

[13] D. Guetta, M. Spada, E. Waxman, Astrophys. J. 559 (2001) 101. astro-ph/0102487]. 
[14] IceCube Collaboration, H. Kolanoski, talk given at ICRC 2011, Bejing, China.

[15] OPERA Collaboration, R. Acquafredda et al., New J. Phys. 8 (2006) 303;

OPERA Collaboration, R. Acquafredda et al., JINST 4 (2009) P04018;

OPERA Collaboration, N. Agafonova et al.,JINST 4 (2009) P06020.

[16] OPERA Collaboration, N. Agafonova et al., Phys. Lett. B 691 (2010) 138.

[17] Ed. K. Elsener, The CERN Neutrino beam to Gran Sasso (Conceptual Technical Design), CERN 98-02, INFN/AE-98/05;

R. Bailey et al., The CERN Neutrino beam to Gran Sasso (NGS) (Addendum to report CERN 98-02, INFN/AE-98/05), CERN-SL/99-034(DI), INFN/AE-99/05. 